\title{
Structure of Nascent Microbial Cellulose I. Effects of Methyl and Methoxy Groups of Direct Blue 1 and 53 on Nascent Microbial Cellulose
}

\author{
Md. Ibrahim H. MondaL ${ }^{\dagger}$ and Akira $\mathrm{KAI}^{\dagger \dagger}$ \\ Department of Industrial Chemistry, Tokyo Metropolitan University, \\ Minami-Ohsawa, Hachioji, Tokyo 192-03, Japan
}

(Received March 18, 1997)

\begin{abstract}
The effects of methyl and methoxy groups in Direct Blue 1 and Direct Blue 53 on the structure of nascent microbial cellulose were characterized by X-ray, solid state ${ }^{13} \mathrm{C}$ NMR and deuteration-IR measurements. There were no other differences between these two direct dyes, except methyl and methoxy groups. The product obtained from Acetobacter-culture in the presence of each Direct Blue 1 and Direct Blue 53 is a crystalline complex composed of a dye and cellulose, and the product has a structure in which the dye molecule is between the cellulose sheets corresponding to the (100) plane of the complex, i.e., (1I0) planes of microbial cellulose. Celluloses regenerated from both products form cellulose II. Although Direct Blue 1 contains two methoxy groups and Direct Blue 53 contains two methyl groups, different effects of these groups on the structure of nascent microbial cellulose were not identified.

KEY WORDS Acetobacter xylinum / Microbial Cellulose / Dye-Cellulose Complex / Cellulose Sheet / Direct Blue 1 / Direct Blue 53 / Regenerated Cellulose /
\end{abstract}

Acetobacter xylinum (A. xylinum), the most studied cellulose producing bacterium, produces cellulose in the form of noncrystalline state which is associated through van der Waals forces and then through hydrogen bonding to form the crystalline cellulose $\mathrm{I} \alpha$ rich fibril. ${ }^{1,2}$ However, until now, the formation mechanism of microbial cellulose (MC) is not clear. Some investigators ${ }^{3-6}$ tried to clarify the mechanism of formation through the alteration procedure by the use of direct dyes and cellulose derivatives. Recently, we reported ${ }^{7-9}$ the structure of nascent MC which interacted with the direct dyes to form the crystalline dye-cellulose complex, when $A$. xylinum was cultured in the presence of a direct dyes. Celluloses regenerated from these dye-cellulose complexes were cellulose II and $\mathrm{IV}_{\mathrm{I}}$ corresponding to the dye used. From our observations, we suggest ${ }^{7-9}$ that the transformation of other cellulose allomorphs during regeneration of the cellulose component in the product is affected mainly by the numbers as well as the position of sulfonate groups in a dye.

The present work reports the effects of methyl and methoxy groups in Direct Blue 1 (DB1) and Direct Blue 53 (DB53) on the structure of nascent MC. The structures of the product, obtained from A. xylinum-culture in the presence of a direct dye, and its regenerated cellulose were characterized by X-ray diffractometer, solid state ${ }^{13} \mathrm{C}$ NMR and deuteration-IR spectroscopies.

\section{EXPERIMENTAL}

\section{Preparation of Samples}

A. xylinum (IFO 13693) was cultured in a $15 \mathrm{~cm}$ diameter petridish containing $100 \mathrm{ml}$ Schramm-Hestrin medium $^{10}(\mathrm{pH} 6.8)$ at $28^{\circ} \mathrm{C}$ for about 3 days in an incubator. From this matured petridish, bacteria were collected and suspended in $120 \mathrm{ml}$ phosphate buffer so- lution. $60 \mathrm{ml}$ of this cellulose-free cell suspension were added to $140 \mathrm{ml}$ of $\mathrm{HS}$ medium $(\mathrm{pH} 7.0)$ containing a dye with concentration of $0.1 \mathrm{wt} \%$ and the mixture was incubated at $28^{\circ} \mathrm{C}$ for about $24 \mathrm{~h}$ in a static conditions. ${ }^{4,8}$ The product sample (product) was collected by centrifuging $(6000 \mathrm{rpm}, 5.15 \mathrm{~g}, 20 \mathrm{~min})$ and washed with distilled water to remove the dye not related to dyeing. These products were preserved at about $3^{\circ} \mathrm{C}$. The direct dyes are DB1 (2,2'-[4,4'-(3,3'-bismethoxy)biphenylenebis(azo)]bis[8-amino-1-hydroxynaphthalene-5,7-disulfonic acid] tetrasodium salt); Aldrich Chemical) and DB53 (2,2'-[4,4'-(3,3'-dimethyl)biphenylenebis(azo)]bis[8-amino-1-hydroxy-naphthalene-5,7-disulfonic acid] tetrasodium salt; Aldrich Chemical) and their chemical structure are shown in Figure 1.

The above samples were subjected to dye extraction by boiling in $70 \mathrm{vol} \%$ aqueous ethanol solutions for $18 \mathrm{~h}$ and fresh aqueous ethanol solution was exchanged every<smiles>COc1cc(-c2ccc(N=Nc3ccc4c([N+](=O)[O-])cc([N+](=O)[O-])c(N)c4c3O)c(OC)c2)ccc1N=Nc1ccc2c(O[Na])cc(OS(N)(=O)=O)c(N)c2c1O</smiles>

\section{Direct Blue 1}

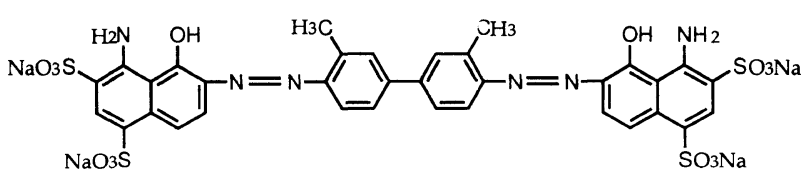

Direct Blue 53

Figure 1. Chemical structure of direct dyes.

${ }^{\dagger}$ Present address: Department of Applied Chemistry and Chemical Technology, Rajshahi University, Rajshahi, Bangladesh.

it To whom correspondence should be addressed. 
$3 \mathrm{~h}$. The dye extracted sample was boiled in $1.0 \mathrm{wt} \%$ aqueous sodium hydroxide solution for $10 \mathrm{~h}$ under $\mathrm{N}_{2}$ atmosphere to make the sample free from proteinous matter. This regenerated cellulose sample was neutralized with $1.0 \mathrm{vol} \%$ aqueous acetic acid solution and subsequently rinsed with distilled water. The sample was preserved as above. ${ }^{4,8}$

To compare the structures of the product and cellulose regenerated from it, control cellulose I and cellulose II were prepared. After washing well with distilled water to remove the medium components, $\mathrm{MC}$ was boiled in $1.0 \mathrm{wt} \%$ aqueous sodium hydroxide solution for $10 \mathrm{~h}$ under $\mathrm{N}_{2}$ atmosphere, and the final sample (cellulose I or MC) was neutralized and rinsed as above. Cellulose II was prepared by mercerization of $\mathrm{MC}$ at $20^{\circ} \mathrm{C}$.

\section{Measurements}

To make clear the structures of product and the cellulose regenerated from it, the following measurements were performed.

An X-ray diffraction diagram of an uniplanar oriented membrane of about $100 \mu \mathrm{m}$ in dry thickness was made by reflection and transmission methods. The membrane was prepared from the preserved product sample and dried on a Teflon plate at room temperature. The diffraction diagrams of an isotropic product sample and its regenerated cellulose were made by transmission. In this method, the sample was compressed as a pellet into the pore of a sample holder of $2 \mathrm{~mm}$ diameter and $1 \mathrm{~mm}$ thickness. The X-ray diffractogram was measured by a MXP $^{18}$ diffractometer (MAC Science) with Ni-filtered $\mathrm{Cu}-K_{\alpha}$ radiation. The measurement conditions were as follows: divergence slit, $1.0 \mathrm{~mm}$; receiving slit, $0.15 \mathrm{~mm}$; scanning speed, $4.0^{\circ} \mathrm{min}^{-1}$; X-ray radiation, $40 \mathrm{kV}, 200$ $\mathrm{mA}$.

Solid state ${ }^{13} \mathrm{C}$ NMR experiments were performed at room temperature on a JEOL JNM EX 270 spectrometer operated at $6.35 \mathrm{~T}$, as described elsewhere. ${ }^{7,8}$ The MAS rates were $5.0-5.5 \mathrm{kHz}$, and chemical shifts relative to tetramethylsilane were determined using the crystalline peak at $17.3 \mathrm{ppm}$ of hexamethyl benzene as the external standard. The non-dried samples were packed in a MAS rotor with an O-ring sealed to avoid loss of water during NMR measurement. The spectrum of the crystalline component was measured by taking NMR signals after relaxation of noncrystalline component. Therefore, in order to determine the crystalline components of control and regenerated celluloses, signals obtained after $\tau=50$ were measured by a T1CP pulse sequence ${ }^{11}$ with $\mathrm{CP}$ as the spectrum of the crystalline component.

To obtain IR spectra of the sample, a membrane of about $10 \mu \mathrm{m}$ in dry thickness was prepared from the non-dried preserved samples, and dried on a Teflon plate. The deuteration method of the sample was performed as described in the previous paper. ${ }^{12,13}$ The sample membrane was set inside a glass cell so that the IR beam was perpendicular to the membrane surface. The glass cell with sample was dried under reduced pressure $\left(10^{-3}\right.$ Torr) for $3 \mathrm{~h}$, and vapour phase deuteration of the sample was performed by $\mathrm{D}_{2} \mathrm{O}(99.8 \%$; Aldrich Chemical) for a given time at room temperature. It was again dried under reduced pressure, and the IR spectrum was measured. The FT-IR system 800 (Nicolet) was used

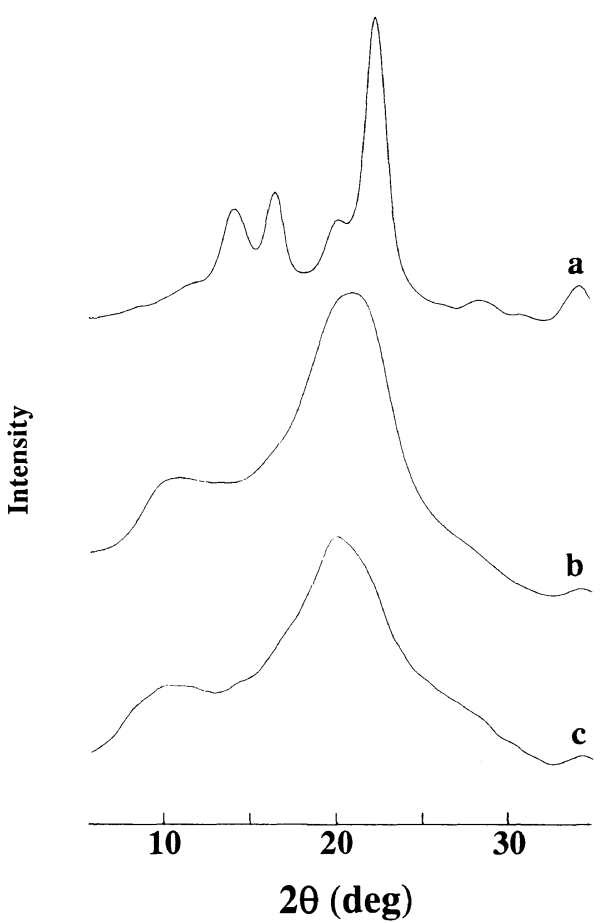

Figure 2. X-Ray diffractograms of microbial cellulose and the product samples from Acetobacter-culture in the presence of a dye of 0.1 wt \%. a, MC; b, DB1 product; c, DB53 product.

Table I. X-Ray diffraction angles and $d$-spacing of products

\begin{tabular}{lccccc}
\hline Sample & \multicolumn{5}{c}{$\begin{array}{c}2 \theta / \mathrm{deg} \\
(d \text {-spacing in } \AA)\end{array}$} \\
\hline MC & - & $14.5(\mathrm{w})$ & $16.8(\mathrm{w})$ & $20.4(\mathrm{w})$ & $22.7(\mathrm{~s})$ \\
& & $(6.12)$ & $(5.28)$ & $(4.35)$ & $(3.92)$ \\
DB1 & $10.9(\mathrm{vw})$ & - & - & $21.1(\mathrm{~s})$ \\
& $(8.09)$ & & & $(4.17)$ \\
DB53 & $10.3(\mathrm{w})$ & - & - & $20.2(\mathrm{~s})$ \\
& $(8.55)$ & & & $(4.44)$ \\
\end{tabular}

for the IR spectroscopy. The amounts of OH and OD groups were calculated as in the previous paper. ${ }^{7}$

\section{RESULTS AND DISCUSSION}

\section{Structure of Product Samples}

$\mathrm{X}$-Ray diffraction diagrams of an isotropic samples of $\mathrm{MC}$ and product samples from Acetobacter-culture in the presence of each DB1 and DB53 (DB1 and DB53 products) are shown in Figure 2. In the diffraction diagrams of DB1 and DB53 products, there are only two diffraction peaks instead of four peaks in the diagram of MC. The different diffraction diagram of the product samples from that of $\mathrm{MC}$ indicates that the product is formed by interaction of dye and cellulose and that the product is a crystalline complex of the dye and cellulose. The lower angle peaks in the diffractograms appear near $10.3-10.9^{\circ}$ while the higher angle peaks appear near $20.2-21.1^{\circ}$, and $2 \theta$ values of the diffraction angle are listed in Table I. As the $d$-spacing of the lower angle plane becomes broader in the product, there is the possibility of dye inclusion in these planes. The $d$-spacing of higher angle plane becomes narrower.

In Figure 3, the X-ray diffraction diagrams of the uniplanar oriented membranes of MC, DB1, and DB53 
Md. I. H. Mondal and A. KaI

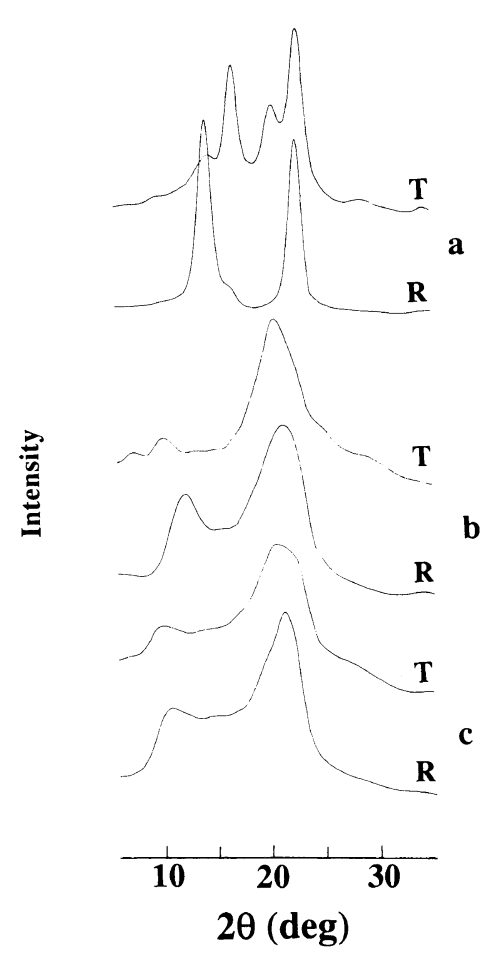

Figure 3. X-Ray diffractograms of oriented membrane of microbial cellulose and product samples measured by transmission $(\mathrm{T})$ and reflection (R) methods. a, MC; b, DB1 product; c, DB53 product.

products are shown. When the DB1 product membrane was measured by reflection method, the intensity of lower angle plane slightly increased to more than that measured by transmission. The diffraction intensity of a higher angle does not change in reflection or transmission. Similar diffraction was observed in the case of DB53 product membrane. Takai et al. ${ }^{14}$ observed the orientation of MC membrane and suggest that if the dif-

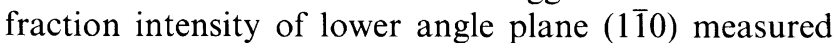
by reflection method is stronger than that measured by transmission method, a lower angle plane is attained preferentially parallel to the surface of the membrane. For vice versa diffraction, in the case of higher angle plane, they suggest that (110) plane is perpendicular to (110) plane. The uniplanar orientation of MC shown in the Figure 3a fully satisfies the Takai et al. concept. However, the uniplanar orientation of DB1 and DB53 product membranes is not similar to that of the MC membrane. The uniplanar orientation of these product membranes is also different to that of DR28 product membrane where a good uniplanar orientation occurs. ${ }^{7}$ From this discussion, it is clear that the lower angle plane of these products is not parallel to the membrane surface and the higher angle plane is not always perpendicular to the membrane surface, i.e., lower angle plane. As the lower angle plane of DB1 and DB53 product membranes shows slightly stronger diffraction intensity measured by reflection than that measured by transmission method, the lower plane has a tendency to become parallel to the surface. This plane orientation of product membranes is similar to that of cellulose II. ${ }^{7}$ The backbone skeletal structures of DR28, DB1 and DB53 are identical, but DB1 and DB53 have more substituent groups on their skeletal structures which may affect the plane orientations of DB1 and DB53 product membranes. There are no

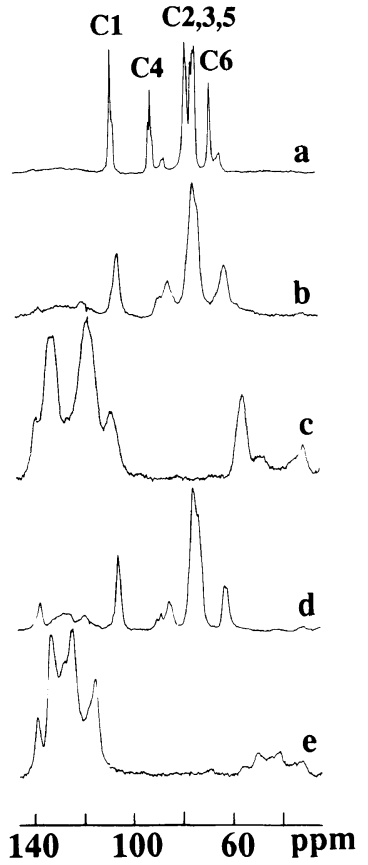

Figure 4. Solid state ${ }^{13} \mathrm{C}$ NMR spectra of the wet $\mathrm{MC}$, wet products and dry direct dyes. a, MC; b, DB1 product; c, DB1 powder; d, DB53 product; e, DB53 powder.

Table II. ${ }^{13} \mathrm{C}$ NMR chemical shifts (ppm) of products

\begin{tabular}{lcccc}
\hline Sample & $\mathrm{C} 1$ & $\mathrm{C} 4$ & $\mathrm{C} 2,3,5$ & $\mathrm{C} 6$ \\
\hline MC & 106.3 & $90.2,84.7$ & $75.9,73.8,72.3$ & $66.5,62.5$ \\
DB1 & 105.2 & -84.7 & $75.2-62.4$ \\
DB53 & 105.6 & -85.0 & $75.5-$ & - \\
\hline
\end{tabular}

differences between the uniplanar orientation of DB1 and DB53 product membranes. These suggest that the uncommon methyl and methoxy groups have no any effect on the uniplanar orientation.

Figure 4 shows the solid state ${ }^{13} \mathrm{C}$ NMR spectra of $\mathrm{MC}, \mathrm{DB} 1$, and DB53 products and their corresponding dye powders. It is apparent from the Figure 4 that no resonance lines of DB1 and DB53 powder overlap the lines of DB1 and DB53 products, respectively. Both spectra of the products are very similar, but different from that of MC. All the resonance lines in the spectra of products are broad compared to that of MC. The chemical shifts of $\mathrm{C} 4$ and $\mathrm{C} 6$ resonance lines of DB1 product are at 84.7 and $62.4 \mathrm{ppm}$, respectively, and similarly, those of DB53 product are at 85.0 and $62.9 \mathrm{ppm}$, respectively. The ${ }^{13} \mathrm{C}$ NMR chemical shifts of MC and product samples are listed in Table II. The corresponding chemical shifts of $\mathrm{C} 4$ and $\mathrm{C} 6$ resonance lines of $\mathrm{MC}$ are the noncrystalline region. Therefore, the solid state ${ }^{13} \mathrm{C}$ NMR spectra of product sample suggest that the product is a noncrystalline complex. This ${ }^{13} \mathrm{C}$ NMR result of product samples is in contrast to the previous $\mathrm{X}$-ray results.

The changes in infrared spectra of $\mathrm{MC}, \mathrm{DB} 1$, and DB53 products are shown in Figure 5 and the amounts of nonaccessible $\mathrm{OH}$ groups, and accessible $\mathrm{OH}$ groups that transform to OD groups by vapour phase deuteration at room temperature with $\mathrm{D}_{2} \mathrm{O}$ are listed in Table III. From Figure 5 and Table III, it is clear that 
Table III. Amounts of $\mathrm{OH}$ and $\mathrm{OD}$ bands of the product and $\mathrm{MC}$

\begin{tabular}{lcc}
\hline \multicolumn{1}{c}{ Sample } & OH band $/ \%$ & OD band $/ \%$ \\
\hline MC & 85 & 15 \\
DB product & 36 & 64 \\
DB53 product & 34 & 66 \\
\hline
\end{tabular}

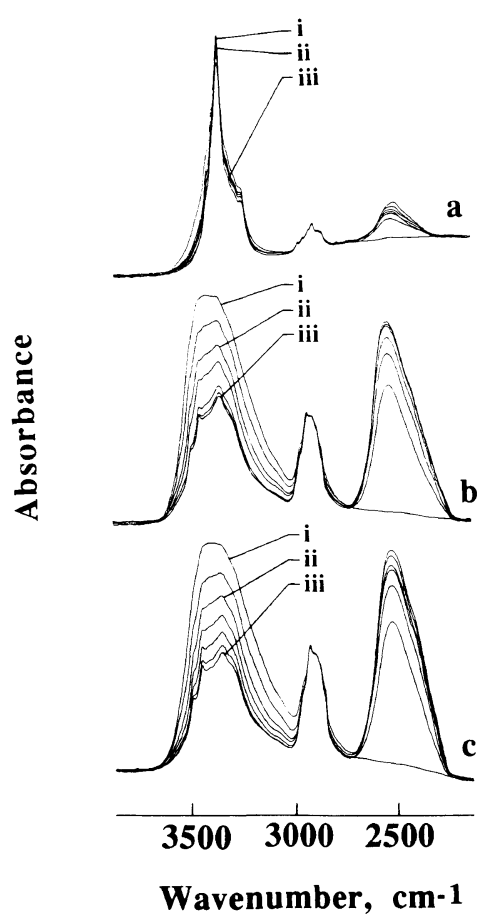

Figure 5. Changes in IR spectra of products by vapour phase deuteration at room temperature. a, MC; b, DB1 product; d, DB53 product. Deuteration time (min): i, 0; ii, 3; iii, 1000.

only about $15 \%$ of accessible $\mathrm{OH}$ groups are present in MC. The amounts of accessible $\mathrm{OH}$ groups in products are four times those of MC. Absorption by $\mathrm{OH}$ groups of the deuterated product samples is near 3479,3439 , and $3342 \mathrm{~cm}^{-1}$ which are the close absorptions of intra- and intermolecular hydrogen bonding of cellulose II (mercerized MC). ${ }^{12}$ These spectra of $\mathrm{OH}$ bands suggest that nonaccessible $\mathrm{OH}$ groups in products are of cellulose II. There are no differences in the two deuterated spectra.

The absorptions by intramolecular hydrogen bonding OH groups of DB1 and DB53 products was near 3479 and $3439 \mathrm{~cm}^{-1}$, respectively, instead of 3485 and $3443 \mathrm{~cm}^{-112}$ of cellulose II. This means the intramolecular hydrogen bonding in the products may be somewhat stronger than that of cellulose II. Absorption by intermolecular hydrogen bonding $\mathrm{OH}$ groups of products was near $3342 \mathrm{~cm}^{-1}$ instead of $3340 \mathrm{~cm}^{-1}$ of cellulose II. Furthermore, the absorption by intermolecular hydrogen bonding $\mathrm{OH}$ groups of cellulose II as a shoulder near $3174 \mathrm{~cm}^{-1}$ was not observed in the product samples. Although the DB1 and DB53 products contains 36 and $34 \%$ nonaccessible $\mathrm{OH}$ groups and the spectral patterns are similar to cellulose II, the intermolecular hydrogen bonding between the cellulose sheets or chains in the products are not strong like cellulose II. A. xylinum normally produces cellulose I.

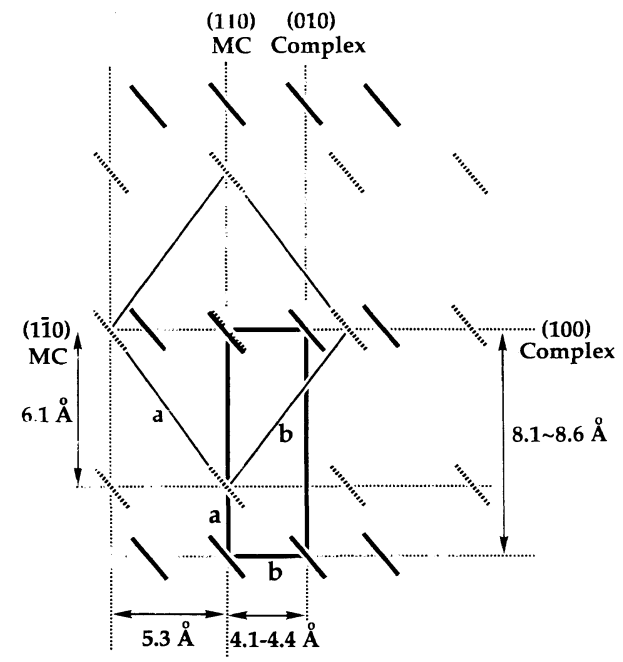

Figure 6. Generalized model structures of microbial cellulose and dye-cellulose complex. Resulting from X-ray diagrams of the product samples, the dye molecule seems to be included between (100) planes in the complex or $(1 \overline{1} 0)$ planes of microbial cellulose. IIIII and configuration of cellulose chains in cellulose I and complex, respectively.

But the nonaccessible $\mathrm{OH}$ group in the products shows the spectra of cellulose II and this conformational change of a cellulose chain in the cellulose component is affected by the dyes. As the spectra of the nonaccessible $\mathrm{OH}$ groups in the products by deuteration is cellulose II, the product is first formed by the interaction of dye and nascent $\mathrm{MC}$ and then, some dye molecules from the product are washed off during rinsing. The portion of the product from where the dye molecules washed off is crystallized to cellulose II through the conformational change. Due to the presence of cellulose II component in the product, the uniplanar orientation of these products is very poor compared to that of MC or DR2 28 product. $^{7,8}$

If the dye molecules is included between the cellulose sheets, the structure of the product can easily be explained by the model shown in Figure 6. From Figure 6 it is clear that the $d$-spacing of the complex component in the product become broad as $8.1-8.6 \AA$ instead of $6.1 \AA$ of MC. Therefore, the dye molecule is included between the

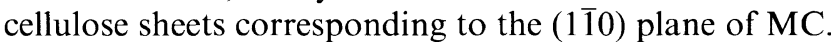
Due to the inclusion of dye between the cellulose sheets, intermolecular hydrogen bonding between the $(020)$ and (110) planes of MC is hindered. If by any means intermolecular hydrogen bonding occurs, the product fails to show good uniplanar orientation. As the spacing of (100) plane of product become broad by loss of intermolecular hydrogen bonding, the van der Waals forces attract the cellulose sheets corresponding to (010) plane each other. Due to the strong attraction between the cellulose sheets, the spacing between the (010) plane becomes narrow. If the (020) plane is absent in the unit cell of complex, the higher angle (010) plane is the (110) plane of MC and perpendicular to the (100) plane. From Figure 6, it is apparent that there is no (020) plane of MC in the complex. This strongly suggests that hydrogen bonding between the cellulose sheets corresponding to the (020) plane of MC in the complex is not possible.

If the structure of the complex has the unit cell shown 
Md. I. H. Mondal and A. KaI

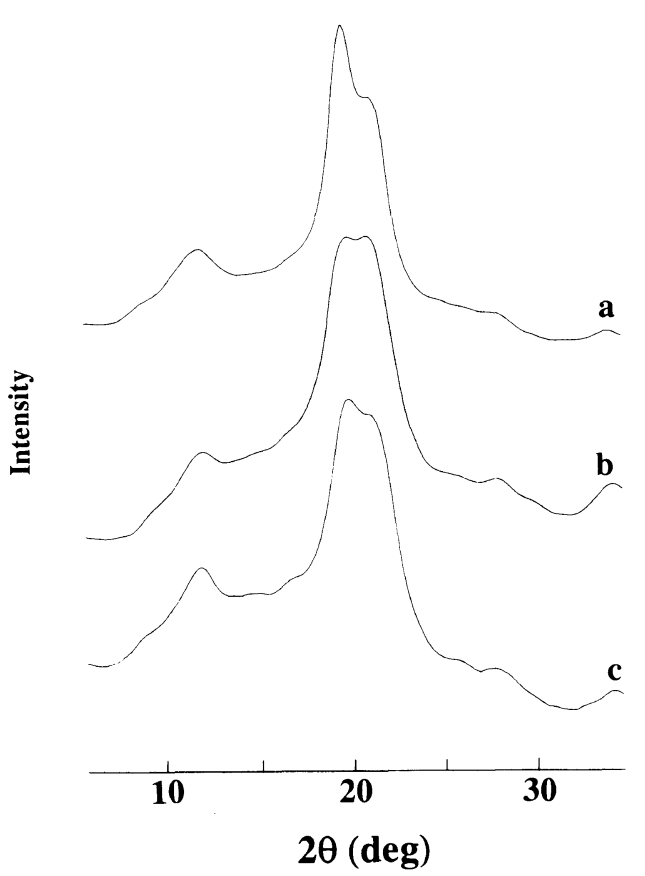

Figure 7. X-Ray diffractograms of celluloses regenerated from the product samples. a, Cellulose II; b, DB1 cellulose; c, DB53 cellulose.

in Figure $6,{ }^{13} \mathrm{C}$ NMR and deuteration-IR results can be explained. From the ${ }^{13} \mathrm{C}$ NMR results, the complex is noncrystalline. This is possible if the dye molecule is hindered to form hydrogen bonding between the cellulose sheets by inclusion between the (100) planes of the complex. The lack of the hydrogen bonding between (100) planes increases the diffusion of $\mathrm{D}_{2} \mathrm{O}$ into the complex. Therefore, the deuteration of accessible $\mathrm{OH}$ groups in the complex occurs easily and as a result, the amount of accessible $\mathrm{OH}$ groups in the complex is always higher than that of MC as well as regenerated celluloses. As the structure of cellulose regenerated from the complex is different from that of $\mathrm{MC}$, a dye affects the conformation of cellulose chain by the direct interaction of cellulose chain and a dye molecule through the formation of a complex. However, if a dye molecule is in a stack state on the surface of a protofibril as Haigler et al. suggested, ${ }^{5}$ it is very difficult to affect the conformation of all cellulose chains by the dye in the product and cellulose regenerated from it. Otherwise, if dyes do not interact with all cellulose chains in the product and the conformational changes do not occur in all the regions, a mixture of $\mathrm{MC}$ and product as well as $\mathrm{MC}$ and regenerated cellulose will be produced.

Structure of Cellulose Regenerated from Product Samples

The X-ray diffraction of cellulose II and celluloses regenerated from $\mathrm{DB} 1$ and DB53 products (DB1 and DB53 celluloses) is shown in Figure 7. DB1 and DB53 celluloses are very similar to cellulose II (mercerized MC). The $2 \theta$ values of (110), (110), and (020) planes of regenerated celluloses are also similar to cellulose II and listed in Table IV. From the diffraction diagrams in Figure 6 and $2 \theta$ in Table IV, it is clear that the DB 1 and DB53 celluloses are cellulose II. The skeletal structures of both the dyes are identical and substituent groups on the skeletal structure are the same, except methyl groups in DB1 and methoxy groups in DB53. But the fact that
Table IV. X-Ray diffraction angles of regenerated cellulose

\begin{tabular}{lccc}
\hline \multicolumn{1}{c}{ Sample } & \multicolumn{3}{c}{$2 \theta / \mathrm{deg}$} \\
& $(1 \overline{1} 0)$ & $(110)$ & $(020)$ \\
\hline Cellulose II & 12.0 & 19.8 & 21.1 \\
DB1 & 12.3 & 20.2 & 21.4 \\
DB53 & 11.8 & 19.9 & 21.6 \\
\hline
\end{tabular}

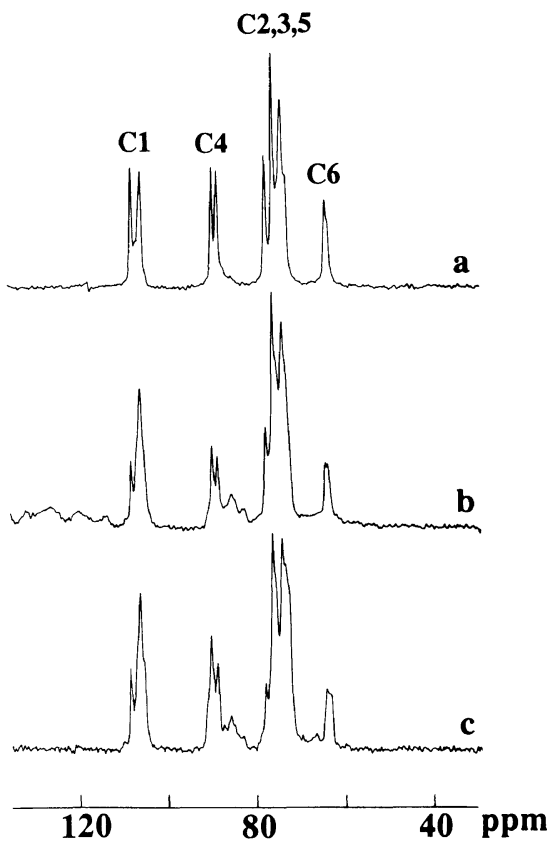

Figure 8. Solid state ${ }^{13} \mathrm{C}$ NMR spectra of crystalline components of the wet cellulose II and wet cellulose regenerated from the products. a, Cellulose II; b, DB1 cellulose; c, DB53 cellulose.

Table V. ${ }^{13} \mathrm{C}$ NMR chemical shifts of crystalline components of regenerated celluloses, cellulose II

\begin{tabular}{|c|c|c|c|c|}
\hline Sample & $\mathrm{Cl}$ & $\mathrm{C} 4$ & $\mathrm{C} 2,3,5$ & C6 \\
\hline Cellulose I & $107.5,105.3$ & $89.3,87.9,-$ & $77.0,75.2,72.9$ & 63.262 .7 \\
\hline DB1 & $107.9,105.7$ & $89.6,88.3,85.3$ & $77.4,75.7,73.4$ & 63.863 .2 \\
\hline DB53 & $107.5,106.2$ & $90.1,88.7,85.6$ & $77.5,75.9,73.8$ & 64.263 .6 \\
\hline
\end{tabular}

diffraction diagrams of DB1 and DB53 celluloses are almost the same means that methyl and methoxy groups have the same effects on nascent MC.

The solid state ${ }^{13} \mathrm{C}$ NMR spectra of crystalline component of DB1 and DB53 celluloses are different from those of their products and are shown in Figure 8. Each single $\mathrm{Cl}$ and $\mathrm{C} 4$ resonance line of products split into two peaks in regenerated celluloses. The $\mathrm{C} 4$ and $\mathrm{C} 6$ resonance lines of DB1 and DB53 celluloses appeare near $87.9-89.3$ and $64.2 \mathrm{ppm}$, respectively. But, the $\mathrm{C} 4$ and C6 resonance lines of products were observed in the noncrystalline region of $\mathrm{MC}$. The chemical shifts of all resonance lines (Table $\mathrm{V}$ ) and splitting of $\mathrm{Cl}$ and $\mathrm{C} 4$ lines indicate that the whole spectra is very similar to cellulose II. Therefore, the DB1 and DB53 celluloses would appear to be cellulose II. These results of regenerated celluloses strongly support the X-ray measurement results. The intensity of the down field peak of $\mathrm{C} 1$ lines is lower than that of upfield peaks. The hydrogen bonding in these regenerated celluloses are thus 


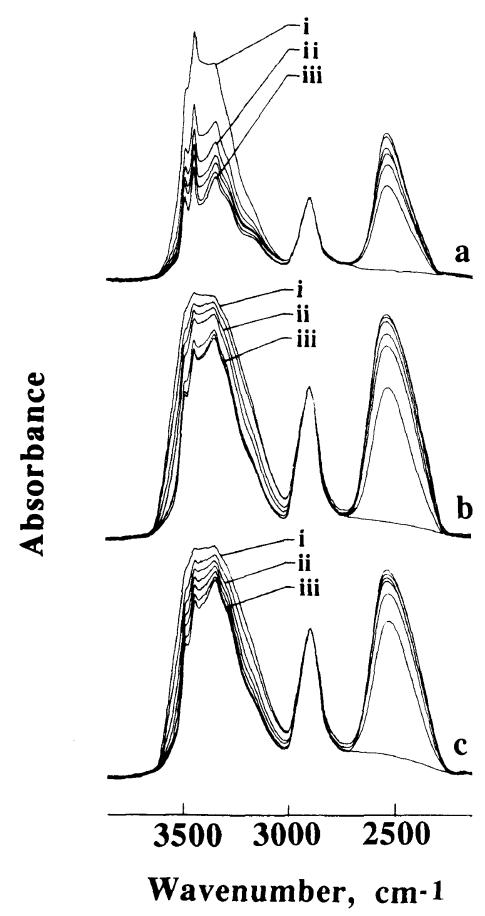

Figure 9. Change in IR spectra of celluloses regenerated from the product samples by vapor phase deuteration at room temperature. a Cellulose II; b, DB1 cellulose; c, DB53 cellulose. Deuteration time (min): i, 0 ; ii, 3; iii, 1000.

Table VI. Amounts of $\mathrm{OH}$ and $\mathrm{OD}$ groups of regenerated cellulose

\begin{tabular}{lcc}
\hline \multicolumn{1}{c}{ Sample } & OH band $/ \%$ & OD band $/ \%$ \\
\hline Cellulose II & 45 & 55 \\
DB1 cellulose & 47 & 53 \\
DB53 cellulose & 48 & 52 \\
\hline
\end{tabular}

not similar to that of cellulose II. The $\mathrm{C} 1$ resonance line is also different from that of DR75 and DR79 celluloses where downfield and upfield peaks intensities are similar to those of cellulose II. ${ }^{9,15}$ The weak downfield peak of $\mathrm{C} 1$ lines indicates that the hydrogen bonding in DB1 and DB53 celluloses is not so strong like cellulose II. ${ }^{9,15}$ The peaks near $85.0 \mathrm{ppm}$ of DB1 and DB53 celluloses are the noncrystalline region of cellulose.

The absorption spectra of $\mathrm{OH}$ bands by vapour phase deuteration at room temperature are shown in Figure 9 and the amounts of nonaccessible and accessible $\mathrm{OH}$ groups $(\mathrm{OH}$ groups that is deuterated to $\mathrm{OD}$ groups ) are listed in Table VI. The absorption by $\mathrm{OH}$ groups after deuteration are the nonaccessible $\mathrm{OH}$ groups, which show the crystal types of regenerated celluloses. The absorption by intramolecular hydrogen bonding of DB1 and DB53 celluloses is at 3483 and $3440 \mathrm{~cm}^{-1}$, respectively, which are $2 \mathrm{~cm}^{-1}$ from $3385 \mathrm{~cm}^{-1}$ and $3 \mathrm{~cm}^{-1}$ from $3443 \mathrm{~cm}^{-1}$ less than that of cellulose II. ${ }^{12}$ This means that the intramolecular hydrogen bonding in DB1 and DB53 celluloses may be somewhat stronger than that of cellulose II, but somewhat weaker than that of DB1 and DB53 products. The absorption by intermolecular hydrogen bonding of DB1 and DB53 celluloses are at 3342 and $3179 \mathrm{~cm}^{-1}$, respectively, whereas that of cellulose II is at 3340 and $3174 \mathrm{~cm}^{-1}$. The absorption of regenerated celluloses near $3179 \mathrm{~cm}^{-1}$ was not observed in the $\mathrm{OH}$ band of the products. Although the celluloses regenerated from DB1 and DB53 products are cellulose II, intermolecular bonding, hindered by the dyes due to their inclusion between the cellulose sheets in the product, developed during regeneration and drying is a little weaker than that of cellulose II, but stronger than that in the products. The increase of the amount of nonaccessible $\mathrm{OH}$ groups in DB1 and DB53 celluloses to 47 and $48 \%$ supports the developement of hydrogen bonding. The intra- and intermolecular hydrogen bonding absorption by $\mathrm{OH}$ groups of DB1 and DB53 celluloses is the same and their absorption spectra are also similar, suggesting that both the dyes have similar effect on the regenerated celluloses. Thus, the celluloses regenerated from DB1 and DB53 products are cellulose II and both possesses similar fine structure, this fine structure are somewhat different from that of cellulose II (mercerized MC).

\section{CONCLUSION}

The effects of methyl and methoxy groups in Direct Blue 1 and Direct Blue 53 on the structure of nascent microbial cellulose were studied by X-ray, solid state ${ }^{13} \mathrm{C}$ NMR and deuteration-IR measurements. The product obtained from Acetobacter-culture in the presence of Direct Blue 1 or Direct Blue 53 is a crystalline complex composed of a dye and cellulose, and the product structure is such that the dye molecule is included between the cellulose sheets corresponding to (100) plane of the complex, i.e., (110) planes of microbial cellulose. Celluloses regenerated from the products form cellulose II. Although Direct Blue 1 contains two methoxy groups and Direct Blue 53 contains two methyl groups, different effects of these groups on the structure of nascent microbial cellulose were not found.

\section{REFERENCES}

1. A. Kai and T. Koseki, Makromol. Chem., 186, 2609 (1985).

2. S. K. Cousins and R. M. Brown, Jr., Polymer, 36, 3885 (1995).

3. A. Kai, P. Xu, F. Horii, and S. Hu, Polymer, 35, 75 (1994).

4. A. Kai, H. Kido, and N. Ishida, Chem. Lett., 949 (1990).

5. C. H. Haigler and H. Chanzy, J. Ultrastruct. Mol. Struct. Res., 98, 299 (1988).

6. R. M. Brown, Jr., C. H. Haigler, J. Suttie, A. R. White, E. Roberts, C. Smith, T. Itoh, and K. Copper, Appl. Polym. Symp., 37, 33 (1983).

7. A. Kai and Md. I. H. Mondal, Int. J. Biol. Macromol., 20, 221 (1997).

8. A. Kai and Md. I. H. Mondal, in "Biomedical Functions and Biotechnology of Natural and Artificial Polymers," M. Yalpani, Ed., ATL Press, Inc., Science Publishers, Shrewsbury, MA, 1996, p 77 .

9. Md. I. H. Mondal and A. Kai, Int. J. Biol. Macromol., in press.

10. S. Hestrin and M. Schramm, Biochem. J., 58, 345 (1954).

11. D. A. Torchia, J. Magn. Reson., 30, 613 (1978).

12. S. Okajima and A. Kai, J. Polym. Sci., A-1, 6, 2801 (1968).

13. A. Kai and P. $\mathrm{Xu}$, in "Cellulosics: Chemical, Biochemical and Material Aspects," (J. F. Kennedy, G. O. Phillips, and P. A. Williams, Ed., Ellis Horwood, London, 1993, p 2325.

14. M. Takai, Y. Tsuta, J, Hayashi, and S. Watanabe, Polymer J., 7, 157 (1975).

15. F. Horii, A. Hirai, and R. Kitamaru, Polym. Bull., 8, 163 (1982). 